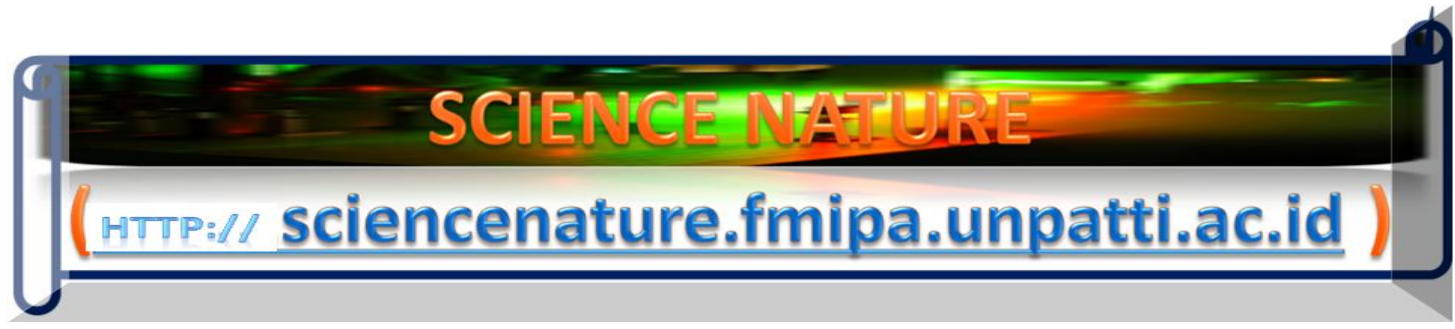

Science Nature 1(1), pp.033-041 (2018)

e-ISSN 2654-6264

DOI: https://doi.org/10.30598/SNVol1Iss1pp033-041year2018

\title{
The Least Number of Intersection Points in Currency Fluctuation and Well- Approximating Line Graph under Constraints of the Elliott Wave Principle
}

\author{
Naoyasu Kita \\ Faculty of Advanced Science and Technology, Kumamoto University \\ Taisei Matsukuma \\ Graduate School of Science and Technology, Kumamoto University
}

Received : September 25, 2018

Revised : October 21, 2018

Accepted : October 22, 2018

Published : October 24, 2018

Copyright @ All rights are reserved by Naoyasu Kita \& Taisei Matsukuma

Corresponding author: "Email: nkita@kumamoto-u.ac.jp 


\section{Abstract}

Recently producing profit by exchanging currency of one country with that of the other has been conducted among traders. This activity is called "FX" - the abbreviation of Foreign eXchange. The fluctuation of the price of currency is caused by the psychology of traders so that it apparently varies at random. However, after so many traders take part in $\mathbf{F X}$, the trend of currency expectedly shows several patterns and is said to be subject to a certain principle which was firstly suggested by R.N.Elliott (1871-1948). In our recent research, we have tried to construct an algorithm relying on this Elliott wave principle and establish an automated transaction of $\mathbf{F X}$ by a computer. One of the aims of this PC-algorithm is to determine the line graph which well-approximates a real currency fluctuation under the constraints of the Elliott principle. In this paper, we will survey how to construct an algorithm for well-approximating line graph, and theoretically discuss the least number of intersection points in currency fluctuation and the well-approximating line graph, which enhances the validity of the algorithm.

Keywords: FX, well-approximating line graph, scale invariant squared error, constrained optimization, method of penalty function, fastest descent method.

\section{ARTICLE}

\section{Introduction}

Today, the FX (initial letters of "Foreign eXchange") is of interest among many finance traders

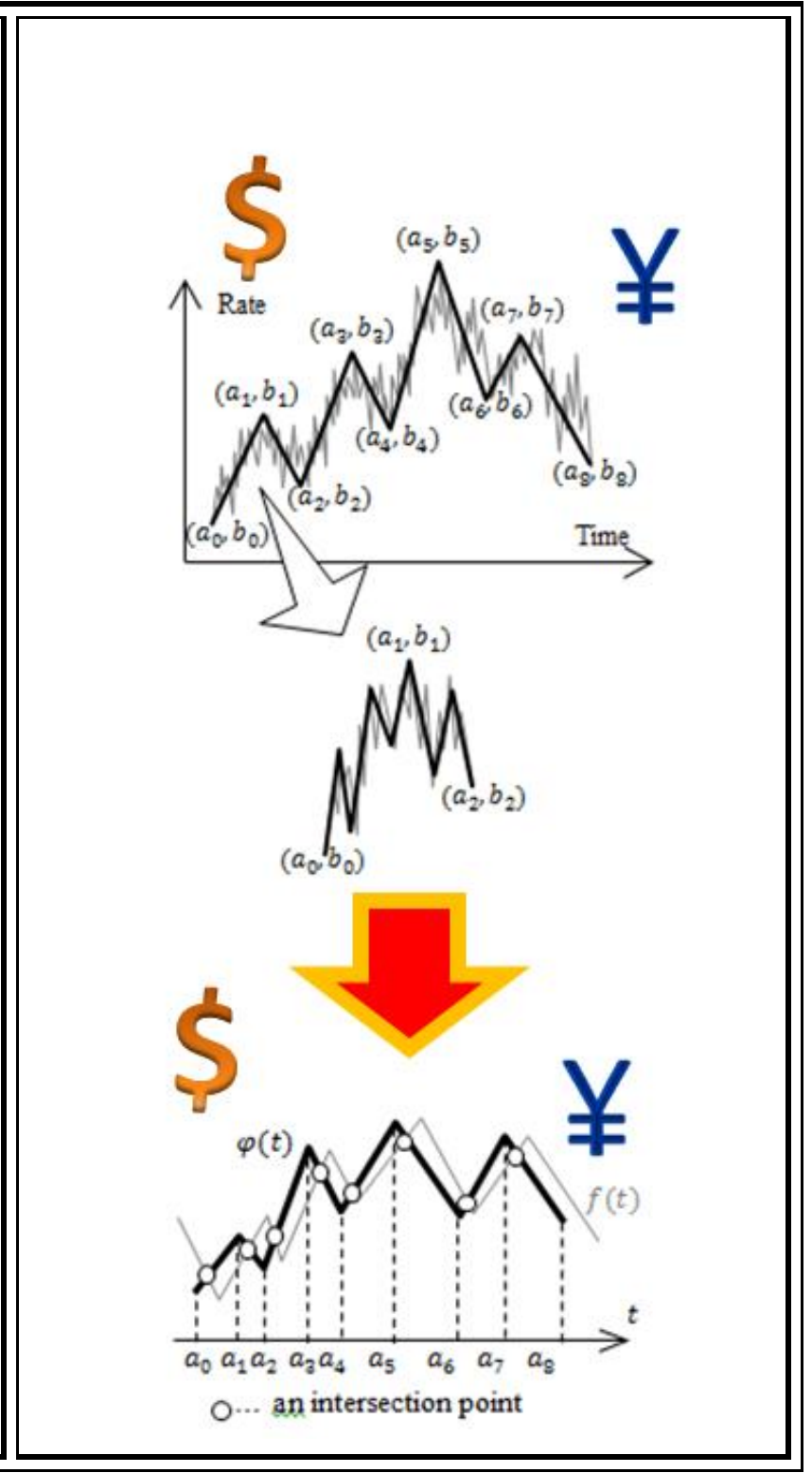

who want to produce profit by making use of fluctuations of exchange rate of foreign currencies. A normal way of obtaining profit is, for example, buying $\$ 100$ (100 US dollers) for $¥ 10,000$ (10,000 JP yen) at one time, and selling the US dollers when $\$ 1=\backslash 110$ : the exchange rate of JPY/USD. Then one has succeeded in making profit of $¥ 110 \times 100-¥ 10,000=$ $¥ 1,000$. The fluctuations of exchange rate is described 
as a randomly bended curve (see Fig.1.1), and so the mathematical forecast has been usually accomplished by means of stochastic theory.

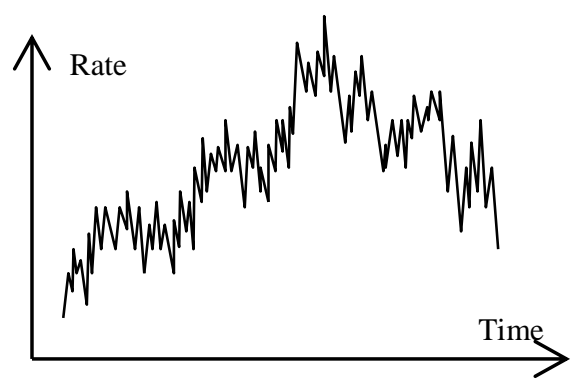

Figure 1.1. Fluctuation of Exchange Rate.

But, in stochastic theory, the observation of expectation values among huge number of trials are often at the core of the analysis. This means that the forecast depending only on the stochastic theory sometimes fails, and when one wants to make profit, he/she must conduct a large number of transactions.

If there is a certain principle or law in the complicatedly fluctuated trends of currencies, we may produce profit more efficiently and in less number of transactions. The Elliott wave principle of our interest may fulfill such desire.

\subsection{Elliott Wave Principle}

Ralph Nelson Elliott (1871-1948) [1,2], a professional accountant, discovered an empirical principle hidden in the complicated motion of market prices [3]. He proposed that market prices progress in specific patterns, which is today called "Elliott waves", or simply "waves". The trend of currency is estimated by a simplified line graph $\varphi(t)$ [4,6], where $\varphi(t)$ is a function of graph linearly connecting 9 points $\left(a_{j}, b_{j}\right) \in R^{2}(j=0,1, \ldots, 8) \quad$ with $a_{0}<a_{1}<\cdots<a_{8}$. Of course, $\varphi\left(a_{j}\right)=b_{j}$. If $1 \leq j \leq 5$, the line segment described by $\varphi(t)$ for $t \in\left[a_{j-1}, a_{j}\right]$ is called "wave- $j$ ", and if $j=6,7,8$, the line segment described by $\varphi(t)$ for $t \in\left[a_{j-1}\right.$, $a_{j}$ ] is called "wave-A", "wave-B" and "wave-C" respectively (See Fig.1.2). The typical Elliott wave consists of these 8 trends described by wave- 1 to wave-C. The number-indexed waves like wave- 1 to wave-5 appear in the increasing trend of rate, and the letter-indexed waves like wave-A to wave-C appear in the decreasing trend of rate. In $[\mathbf{4 , 6}]$, there is no way to identify the line graph shown, and the analysis of the trend is possibly fallen into subjective one. In this paper, we will propose a mathematical construction of $\varphi(t)$, the detail of which will be discussed later.

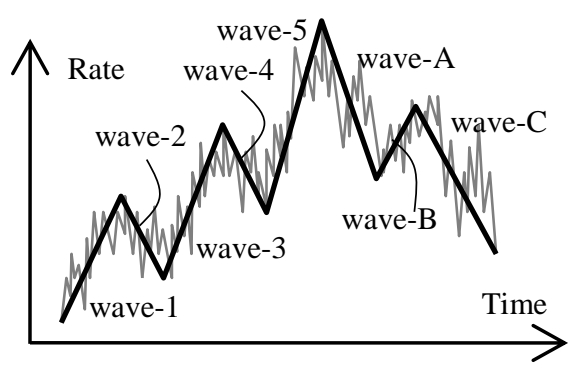

Figure 1.2. Line Graph $\varphi(t)$.

In addition, the characters of a typical Elliott wave are presented by the next three patterns (See Fig.1.3). These patterns are the mathematical representation of the Elliott wave principle $[7,8]$.

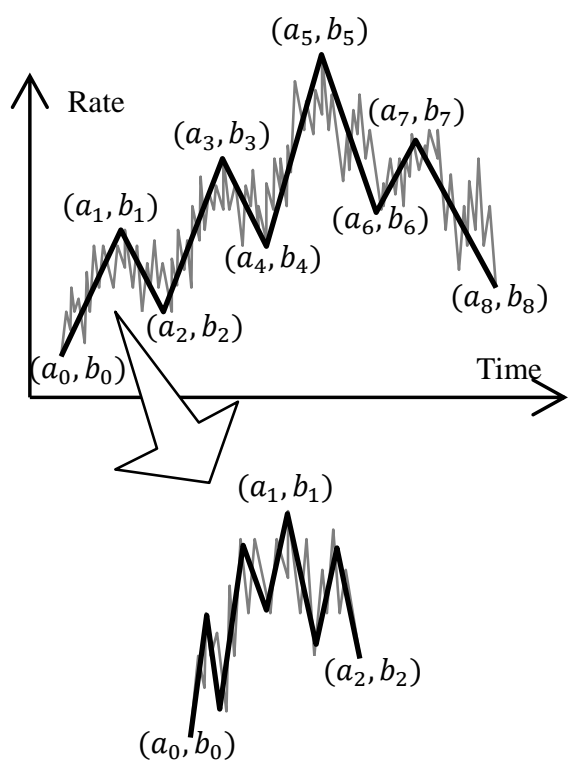

Figure 1.3. Characters of Elliott Wave.

(pattern 1 : structure on high and low) The $b_{j}$ 's satisfy 


$$
\begin{aligned}
& b_{0}<b_{1}, b_{1}>b_{2}, b_{2}<b_{3}, \\
& b_{3}>b_{4}, b_{4}<b_{5}, b_{5}>b_{6}, \\
& b_{6}<b_{7}, b_{7}>b_{8} .
\end{aligned}
$$

(pattern 2 : structure on ratio) The relationship of wave- 1 and wave- 2 is

$$
\left(b_{1}-b_{0}\right) \times 0.618>b_{1}-b_{2} \text {. }
$$

The relationship of wave- 1 and wave- 3 is

$$
\left(b_{1}-b_{0}\right) \times 1.618=b_{3}-b_{2} \text {. }
$$

The relationship of wave- 3 and wave- 4 is

$$
\left(b_{3}-b_{2}\right) \times 0.382>b_{3}-b_{4} \text {. }
$$

The relationship of wave- 3 and wave- 5 is

$$
\left(b_{3}-b_{2}\right) \times 1.000>b_{5}-b_{4} \text {. }
$$

The relationship of wave-A and wave- $\mathrm{C}$ is

$$
\left(b_{5}-b_{6}\right) \times 1.618=b_{7}-b_{8} \text {. }
$$

The detail on the ratio is also written in [7].

(pattern 3 : self-similar structure) By observing the combination of wave-1 and wave-2 closely, the similar but smaller patterns as in pattern1 and pattern2 are found again. The same is true for the combinations of wave- 3,4 and wave-B,C.

We are taking the character described in pattern 3 above so important, because a small Elliott wave (called "Elliott sub-wave", or simply "sub-wave") forecasts the coming of next lager Elliott wave. This implies that detecting an Elliott sub-wave informs a good opportunity of buying in or selling out foreign currencies.

Since financial market cycles are generated by the collective psychology of investors, there are some criticism against the Elliott wave principle, insisting that group psychology cannot be subject to such simple mathematical constraints $[\mathbf{1 , 5}]$. In this paper, however, the validity of the Elliott wave principle won't be discussed. This paper is aiming at two targets. One aim is to introduce the idea on how to construct the approximating line graph $\varphi(t)$ by which a portion of Elliott sub-wave is detected in a complicated currency fluctuation. The FX traders who apply the Elliott wave principle have so far detected the sub-wave, relying on their own eyes and feelings.
But we want to propose more efficient an objective way of detection with PC. The other aim is to provide a mathematical property of well-approximation $\varphi(t)$, which is helpful to make sure whether an Elliott sub-wave arises and to check the validity of PC-algorithm.

\subsection{Construction of $\varphi(t)$}

Let us here explain how to construct a line graph $\varphi(t)$ which is a good approximation of a currency fluctuation and will possibly suggest the inception of an Elliott sub-wave. Let $f(t)$ be a continuous function on $R$ which indicates the currency fluctuation. To obtain a good approximation of the currency fluctuation with a line graph, roughly speaking, the minimal value of the objective function:

$\operatorname{SEf}(\vec{a}, \vec{b})=\frac{\int_{a_{0}}^{a_{8}}(f(t)-\varphi(t))^{2} d t}{\int_{a_{0}}^{a_{8}} f(t)^{2} d t}$

is considered, where $\vec{a}=\left(a_{0}, a_{1}, \ldots, a_{8}\right) \in R^{9}$ and $\vec{b}=\left(b_{0}, b_{1}, \ldots, b_{8}\right) \in R^{9} . \boldsymbol{S E f}(\vec{a}, \vec{b})$ plays a role of a squared error of the line graph $\varphi(t)$ from the given currency fluctuation $f(t)$. The square integral $\int_{a_{0}}^{a_{8}} f(t)^{2} d t$ in the devisor of (1.2.1) is taken into account for the next two reasons:

(reason 1) If the objective function was simply given by a square integral $\int_{a_{0}}^{a_{8}}(f(t)-\varphi(t))^{2} d t$, the minimization might possibly take place when $a_{0}=a_{8}$, which means that the both ends of the interval collapse. To avoid such a collapse of the interval, the square integral is divided with $\int_{a_{0}}^{a_{8}} f(t)^{2} d t$.

(reason 2 : scale-invariance) In $\mathbf{F X}$ transaction, we may treat many kinds of currencies, i.e., not only US dollar-JP yen $(1 \mathrm{USD} \approx 100 \mathrm{JPY}$ ) but also Indonesian Rupiah-JP yen $(1 \mathrm{IDR} \approx 0.01 \mathrm{JPY})$ etc. The decimal order of currency rate largely depends on which currencies we will treat. It is mathematically translated 
as the size of $f(t)$ and $\varphi(t)$ remarkably depends on currencies. On the other hand, the period of our observation, i.e., the scale of the time interval $\left[a_{0}, a_{8}\right]$ which we want to observe may change from time to time, and furthermore the unit of time may change at each situation, e.g., we may treat minute, hour, day or week for time-unit. Since the integration of substitution yields

$$
\begin{aligned}
& \boldsymbol{S E f}_{\lambda, \mu}(\mu \vec{a}, \lambda \vec{b})=\frac{\int_{\mu a_{0}}^{\mu a_{8}}\left(f_{\lambda, \mu}(t)-\varphi_{\lambda, \mu}(t)\right)^{2} d t}{\int_{\mu a_{0}}^{\mu a_{8}} f_{\lambda, \mu}(t)^{2} d t} \\
& =\frac{\int_{a_{0}}^{a_{8}}(f(t)-\varphi(t))^{2} d t}{\int_{a_{0}}^{a_{8}} f(t)^{2} d t} \\
& =\boldsymbol{S E} \boldsymbol{f} \quad(\vec{a}, \vec{b})
\end{aligned}
$$

for rescaled functions $f_{\lambda, \mu}(t)=\lambda f(t / \mu)$ and $\varphi_{\lambda, \mu}(t)=\lambda \varphi(t / \mu)$ defined on $\left[\mu a_{0}, \mu a_{8}\right]$ with $\lambda, \mu>0$, the squared error $\boldsymbol{S E} \boldsymbol{f}(\vec{a}, \vec{b})$ is not affected by the scale change. Hence, it may possibly become a universal index indicating the accuracy of the approximation at any transactions of currencies and under time-periods.

\subsection{Constrained Optimization}

We are trying to find $\vec{a} \in R^{9}$ and $\vec{b} \in R^{9}$ which minimizes $\operatorname{SE} \boldsymbol{f}(\vec{a}, \vec{b})$ in $(1.2 .1)$, but they are constrained in (1.1.1) - (1.1.6). It means to detect a portion of Elliott sub-wave in a currency fluctuation described by $f(t)$. Thus we have to apply the method of constrained optimization. Since the constraints (1.1.3) and (1.1.6) in pattern 2 are given by identities, they are too strict when the penalty function method [2] is applied. Replacing (1.1.3) and (1.1.6) by the conditions of inequality, we employ the following relaxed constraints:

(pattern 2': relaxed structure on ratio) The relationship of wave- 1 and wave- 2 is

$$
\left(b_{1}-b_{0}\right) \times 0.618>b_{1}-b_{2} \text {. }
$$

The relationship of wave- 1 and wave- 3 is

$$
1.600<\frac{b_{3}-b_{2}}{b_{1}-b_{0}}<1.650
$$

The relationship of wave- 3 and wave- 4 is

$$
\left(b_{3}-b_{2}\right) \times 0.382>b_{3}-b_{4} \text {. }
$$

The relationship of wave- 3 and wave- 5 is

$$
\left(b_{3}-b_{2}\right) \times 1.000>b_{5}-b_{4} \text {. }
$$

The relationship of wave-A and wave- $\mathrm{C}$ is

$$
1.600<\frac{b_{7}-b_{8}}{b_{5}-b_{6}}<1.650 .
$$

In order to minimize $\boldsymbol{S E} \boldsymbol{f}(\vec{a}, \vec{b})$ under the constraints (1.1.1) and (1.3.1) - (1.3.5), the penalty function method is sometimes applied. Namely we consider the modified squared error defined by

$$
\begin{aligned}
\text { MSEf }(\vec{a}, \vec{b})= & \boldsymbol{S} \boldsymbol{E} \boldsymbol{f}(\vec{a}, \vec{b}) \\
& +k P(\vec{b}),
\end{aligned}
$$

where $k>0$ is a constant and the penalty function $P(\vec{b})$ is given by

$$
\begin{aligned}
& P(\vec{b}) \\
& =\left(\frac{b_{0}-b_{1}}{b_{0}}\right)_{+}^{m}+\left(\frac{b_{2}-b_{1}}{b_{0}}\right)_{+}^{m}+\left(\frac{b_{2}-b_{3}}{b_{0}}\right)_{+}^{m} \\
& +\left(\frac{b_{4}-b_{3}}{b_{0}}\right)_{+}^{m}+\left(\frac{b_{4}-b_{5}}{b_{0}}\right)_{+}^{m}+\left(\frac{b_{6}-b_{5}}{b_{0}}\right)_{+}^{m} \\
& +\left(\frac{b_{6}-b_{7}}{b_{0}}\right)_{+}^{m}+\left(\frac{b_{8}-b_{7}}{b_{0}}\right)_{+}^{m}{ }_{+}^{m} \\
& +\left(\frac{b_{1}-b_{2}-0.618\left(b_{1}-b_{0}\right)}{b_{0}}\right)_{+}^{m} \\
& \left.+\left(\frac{1.600\left(b_{1}-b_{0}\right)-b_{3}+b_{2}}{b_{0}}\right)_{+}^{m}\right)_{+} \\
& +\left(\frac{b_{3}-b_{2}-1.650\left(b_{1}-b_{0}\right)}{b_{0}}\right)^{m} \\
& +\left(\frac{b_{3}-b_{4}-0.382\left(b_{3}-b_{2}\right)}{b_{0}}\right)_{+}^{m} \\
& +\left(\frac{1.600\left(b_{5}-b_{6}\right)-b_{7}+b_{8}}{b_{0}}\right)_{+}^{m} \\
& +\left(\frac{b_{7}-b_{8}-1.650\left(b_{5}-b_{6}\right)}{b_{0}}\right)_{+}^{m} .
\end{aligned}
$$

In the above expression, we defined $(x)_{+}=x$ if $x \geq 0$, and $(x)_{+}=0$ if $x<0$. The exponent $m$ is usually taken as 2 or 3 . If $\vec{b}$ satisfies the relaxed structure of ratio (1.3.1) - (1.3.5), then all the terms in the penalty function $P(\vec{b})$ obviously vanishes and 
hence $\operatorname{MSE} \boldsymbol{f}(\vec{a}, \vec{b})=S E_{f}(\vec{a}, \vec{b})$. Also $\operatorname{MSE} \boldsymbol{f}(\vec{a}, \vec{b})$

still admits the scale invariance, i.e.,

$$
\operatorname{MSEf}_{\lambda, \mu}(\mu \vec{a}, \lambda \vec{b})=\operatorname{MSE\boldsymbol {f}}(\vec{a}, \vec{b}) .
$$

The rough idea of the method of penalty function is that, for some large $k>0$, the minimizer $(\vec{a}, \vec{b})$ must satisfy $P(\vec{b}) \approx 0$ so that $k P(\vec{b})$ does not too largely affect the minimal value of $\operatorname{MSE}_{f}(\vec{a}, \vec{b})$. This means that the minimizers of $\operatorname{MSE}_{f}(\vec{a}, \vec{b})$ lies closely to the constraints (1.1.1) and (1.3.1) - (1.3.5).

\subsection{Algorithm for Minimization}

We are going to devise an algorithm to minimize $\operatorname{MSE}_{f}(\vec{a}, \vec{b})$ by applying the fastest descent method.

Note that the gradient:

$$
\begin{aligned}
& \nabla M S E_{f}(\vec{a}, \vec{b}) \\
& \quad=\left(\partial_{a 0}, \ldots, \partial_{a 8}, \partial_{b 0}, \ldots, \partial_{b 8}\right) M S E_{f}(\vec{a}, \vec{b})
\end{aligned}
$$

is directed in such a way that $\operatorname{MSE}_{f}(\vec{a}, \vec{b})$ increases. Then the recursive relation:

$$
\begin{aligned}
& a_{j}^{(n+1)}=a_{j}^{(n)}-s \frac{\partial_{a j} \text { MSEf }\left(\vec{a}^{(n)}, \vec{b}^{(n)}\right)}{\left|\nabla M \boldsymbol{S E} \boldsymbol{f}\left(\vec{a}^{(n)}, \vec{b}^{(n)}\right)\right|}, \\
& b_{j}^{(n+1)}=b_{j}^{(n)}-s \frac{\partial_{b j} \operatorname{MSE} \boldsymbol{f}\left(\vec{a}^{(n)}, \vec{b}^{(n)}\right)}{\left|\nabla M \boldsymbol{S E \boldsymbol { f }}\left(\vec{a}^{(n)}, \vec{b}^{(n)}\right)\right|}
\end{aligned}
$$

for $j=0,1, \ldots, 8$, provides a minimizing sequence of MSEf $(\vec{a}, \vec{b})$, where the coefficient $s>0$ denotes a step length from $\left(\vec{a}^{(n)}, \vec{b}^{(n)}\right)$ to $\left(\vec{a}^{(n+1)}, \vec{b}^{(n+1)}\right)$ and

$$
\begin{aligned}
& \vec{a}^{(n)}=\left(a_{0}^{(n)}, a_{1}^{(n)}, \ldots, a_{8}^{(n)}\right), \\
& \vec{b}^{(n)}=\left(b_{0}^{(n)}, b_{1}^{(n)}, \ldots, b_{8}^{(n)}\right) .
\end{aligned}
$$

Quitting the iterative scheme at somewhat large $n^{*}$, we are allowed to regard $\left(\vec{a}^{\left(n^{*}\right)}, \vec{b}^{\left(n^{*}\right)}\right)$ as a minimizer of $\boldsymbol{M S E \boldsymbol { f }}(\vec{a}, \vec{b})$ and we are able to construct a $\varphi(t)$ by linearly connecting the 9 points $\left(a_{j}^{\left(n^{*}\right)}, b_{j}^{\left(n^{*}\right)}\right)(j=0,1, \ldots, 8)$. After that, we will evaluate $\operatorname{MS} \boldsymbol{E} \boldsymbol{f}\left(\vec{a}^{\left(n^{*}\right)}, \vec{b}^{\left(n^{*}\right)}\right)$. If $M \boldsymbol{S} \boldsymbol{E} \boldsymbol{f}\left(\vec{a}^{\left(n^{*}\right)}, \vec{b}^{\left(n^{*}\right)}\right)$ is smaller than a certain threshold determined by investigating past data of currency trends, then we can decide whether an Elliot sub-wave is now taking place or not. If an Elliott sub-wave progresses, the coming of next large Elliott wave is expected due to pattern 3 .

\section{Mathematical Theorem}

In this chapter, we will prove a geometrical theorem concerning the property of the well-approximation $\varphi(t)$. This mathematical assertion is useful every time we check the validity of the result from a PC-algorithm.

\subsection{Definition of Feasible Set $\&$ Theorem}

A line graph $\varphi(t)$ generated by a pair of $\vec{a}$ and $\vec{b}$ which satisfies pattern 1 (1.1.1) and pattern 2' (1.3.1)

- (1.3.5) is an ideal minimizer of $\operatorname{MSE}_{f}(\vec{a}, \vec{b})$ since it suggests the presence of an Elliott sub-wave. So we want to give a special name to a family of such $\vec{a}, \vec{b}$.

\section{Definition 2.1.1 (the Feasible Set)}

We define the feasible set as

$$
\begin{aligned}
F S=\{ & \left(a_{0}, \ldots, a_{8}, b_{0}, \ldots, b_{8}\right) \in R^{9} \times R^{9} ; \\
& a_{0}<a_{1}<\cdots<a_{8}, \\
& b_{0}, b_{1}, \ldots, b_{8} \text { satisfy pattern } 1 \quad(\mathbf{1 . 1 . 1}) \text { and } \\
& \text { pattern } \left.2^{\prime}(\mathbf{1 . 3 . 1})-(\mathbf{1 . 3 . 5}) .\right\} .
\end{aligned}
$$

The feasible set indicates that the line graph $\varphi(t)$ generated by $(\vec{a}, \vec{b}) \in F S$ satisfies the constraints of Elliott wave principle. Note that the $F S$ is an open subset of $R^{9} \times R^{9}$. The compliment of $F S$, i.e., $R^{9} \times R^{9} \backslash F S$ is called "the forbidden set".

In this paper, we want to discuss some mathematical properties of the minimizer $\varphi(t)$ generated by $(\vec{a}, \vec{b}) \in F S$, because they are very crucial when we decide whether a result of PC-algorithm is valid or not. What we are curious about is the least number of 
the intersection points in the graphs of $f(t)$ and $\varphi(t)$.

\section{Theorem 2.1.2}

Let $f(t)$ be a continuous function on $R$ and take positive values. Furthermore let $(\vec{a}, \vec{b}) \in F S$ and let $\varphi(t)$ (generated by $(\vec{a}, \vec{b}))$ minimize $\operatorname{MSE}_{f}(\vec{a}, \vec{b})$ (or $S E_{f}(\vec{a}, \vec{b})$ ). Then the graphs of $f(t)$ and $\varphi(t)$ admit at least 8 points of intersection in the strip region $a_{0}<t<a_{8}$.

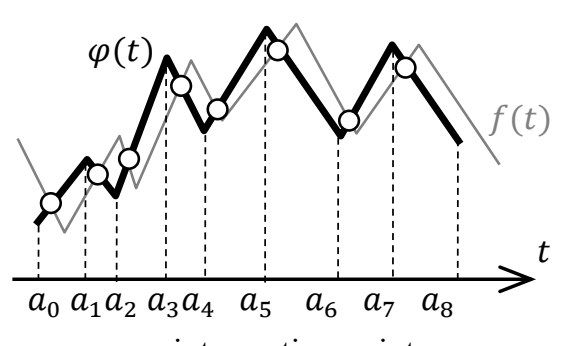

O ... an intersection point

Figure 2.1 Sketch of Theorem 2.1.2.

\subsection{Proof of Theorem 2.1 .2}

Let $\chi(t)$ be a continuous function defined on the closed interval $\left[a_{0}, a_{8}\right]$, the graph of which linearly connects the 9 points $\left(a_{j}, c_{j}\right) \in R^{2}(j=0,1, \ldots, 8)$ for arbitrarily given $c_{j} \in R$. If $(\vec{a}, \vec{b}) \in R^{9} \times R^{9}$ belong to the feasible set and the function $\varphi(t)$ generated by $(\vec{a}, \vec{b})$ admits a minimal value of $\operatorname{MSE} \boldsymbol{f}(\vec{a}, \vec{b})$ (or $\boldsymbol{S E} \boldsymbol{f}(\vec{a}, \vec{b})$ ), then, for any real number $s$ with $|s|$ sufficiently small, $(\vec{a}, \vec{b}+s \vec{c})$ still belongs to the feasible set, where $\vec{c}=\left(c_{0}\right.$, $\left.c_{1}, \ldots, c_{8}\right)$. Furthermore, since $\boldsymbol{S E} \boldsymbol{f}(\vec{a}, \vec{b})$ is assumed to be minimal at present, it follows that

$$
S E_{f}(\vec{a}, \vec{b}) \leq S E_{f}(\vec{a}, \vec{b}+s \vec{c})
$$

holds, where the function $\varphi(t)+s \chi(t)$ is included in the integrand of $\boldsymbol{S} \boldsymbol{E} \boldsymbol{f}(\vec{a}, \vec{b}+s \vec{c})$. By (2.2.1), we see that

$$
\begin{aligned}
& \frac{-2 \int_{a_{0}}^{a_{8}}(f(t)-\varphi(t)) \chi(t) d t}{\int_{a_{0}}^{a_{8}} f(t)^{2} d t} \\
& \quad=\lim _{s \rightarrow+0} \frac{S E_{f}(\vec{a}, \vec{b}+s \vec{c})-S E_{f}(\vec{a}, \vec{b})}{S} \geq 0
\end{aligned}
$$

and

$$
\begin{aligned}
& \frac{-2 \int_{a_{0}}^{a_{8}}(f(t)-\varphi(t)) \chi(t) d t}{\int_{a_{0}}^{a_{8}} f(t)^{2} d t} \\
& =\lim _{s \rightarrow-0} \frac{S E_{f}(\vec{a}, \vec{b}+s \vec{c})-S E_{f}(\vec{a}, \vec{b})}{s} \leq 0 .
\end{aligned}
$$

From these two estimates, it follows that

$$
\int_{a_{0}}^{a_{8}}(f(t)-\varphi(t)) \chi(t) d t=0 .
$$

The integral identity (2.2.2) will play an important role to determine the least number of zeros of $f(t)-\varphi(t)$ later.

The next lemma is the key to complete the proof of Theorem 2.1.2.

\section{Lemma 2.2.1}

Let $k$ denote any natural numbers. Assume that an interval $\left(a_{0}, a_{k}\right)$ is separated by $k$ sub-intervals, and its partition points are $a_{0}<a_{2}<\cdots<a_{k-1}$. Let $g(t)$ be a continuous function on $R$ and possess at most $k-1$ zeros in the interval $\left(a_{0}, a_{k}\right)$. Then there exists a continuous function $\chi(t)$ on $\left[a_{0}, a_{k}\right]$ which satisfies the following two properties:

(1) The graph of $\chi(t)$ linearly connects the points $\left(a_{j}, c_{j}\right) \in R^{2}(j=0,1, \ldots, k-1)$ and $\left(a_{k}, 0\right) \in$ $R^{2}$ for properly determined $c_{j}$ 's.

(2) $\int_{a_{0}}^{a_{k}} g(t) \chi(t) d t \neq 0$.

Proof. It suffices to consider only the case in which all the zeros of $g(t)$ are transverse, i.e., the sign of $g(t)$ alters before and after each zero - if some zeros of $g(t)$ are tangential, they are able to be out of our consideration in this proof. We shall apply the mathematical induction.

(i) The case $k=1$. Nothe that there is no zero in the open interval $\left(a_{0}, a_{1}\right)$. Without loss of 
generality, we can assume that $g(t)>0$. Then it is easy to see that a linear function $\chi(t)=a_{1}-t$ provides $\int_{a_{0}}^{a_{1}} g(t) \chi(t) d t \neq 0$ (See Fig.2.2 (a)).

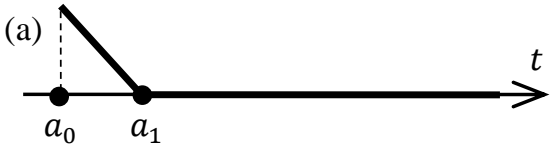

(b)

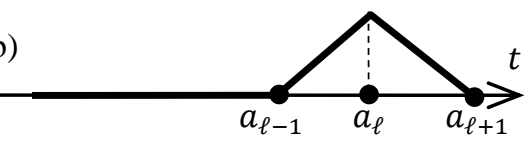

(c)
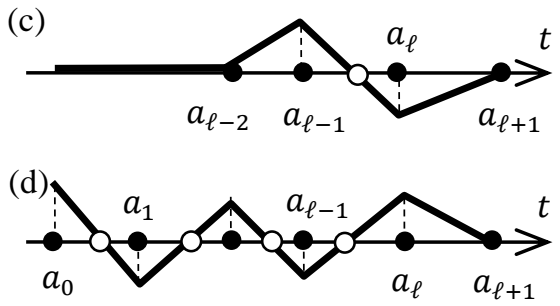

Figure 2.2. Graph of $\chi(t)$.

(ii) The case $k \leq \ell$. We assume that the results in Lemma 2.1 hold. Under this assumption, we are going to consider the case $k=\ell+1$. If there exist some zeros of $g(t)$ in the rightmost sub-interval $\left[a_{\ell}, a_{\ell+1}\right)$, then at most $\ell-1$ zeros are in the sub-interval $\left(a_{0}, a_{\ell}\right)$. By the assumption of the induction, we obtain the desired function $\chi(t)$ on $\left(a_{0}, a_{\ell}\right]$ first, and extend it on $\left(a_{\ell}, a_{\ell+1}\right]$ by $\chi(t)=0$.

Thus it suffices to consider the case $g(t) \neq 0$ on $\left[a_{\ell}, a_{\ell+1}\right)$. If $g(t)$ possesses more than one zero in $\left[a_{\ell-1}, a_{\ell}\right)$, the assumption of the induction still guarantees the presence of the desired $\chi(t)$. On the other hand, if $g(t)$ possesses no zero in $\left[a_{\ell-1}, a_{\ell}\right)$ or one zero at $a_{\ell-1}$, then the desired $\chi(t)$ is obtained by linearly connecting 3 points $\left(a_{\ell-1}, 0\right),\left(a_{\ell}, 1\right)$ and $\left(a_{\ell+1}, 0\right) \in R^{2}$ on $\left[a_{\ell-1}, a_{\ell}\right]$, and $\chi(t)=0$ otherwise (See Fig.2.2(b)). Therefore the remained case is that $g(t)$ possesses only one zero in the sub-interval $\left(a_{\ell-1}, a_{\ell}\right) ; g(t)$ possesses at most $\ell-1$ zeros in $\left(a_{0}, a_{\ell-1}\right)$.

If $g(t)$ possesses more than one zero in $\left[a_{\ell-2}, a_{\ell-1}\right)$, the assumption of the induction guarantees the presence of the desired $\chi(t)$. If $g(t)$ possesses no zero in $\left[a_{\ell-2}, a_{\ell-1}\right)$ or one zero at $a_{\ell-2}$, then the desired $\chi(t)$ is obtained by linearly connecting 4 points $\left(a_{\ell-2}, 0\right)$, $\left(a_{\ell-1}, c_{\ell-1}\right),\left(a_{\ell}, c_{\ell}\right)$ and $\left(a_{\ell+1}, 0\right) \in R^{2}$ on $\left[a_{\ell-1}, a_{\ell}\right]$ where $c_{\ell-1}$ and $c_{\ell}$ are chosen so that the graph of $\chi(t)$ passes the zero in the sub-interval $\left(a_{\ell-1}, a_{\ell}\right), \quad$ and $\quad \chi(t)=0$ otherwise; the graph of $\chi(t)$ partly forms a zigzag shape (See Fig.2.2(c)). Therefore the remained case is that $g(t)$ still possesses only one zero in the sub-interval $\left(a_{\ell-2}, a_{\ell-1}\right) ; g(t)$ possesses at most $\ell-2$ zeros in $\left(a_{0}, a_{\ell-2}\right)$.

By repeating the same argument, the finally remained case is that $g(t)$ admits zeros at each sub-interval $\left(a_{j-1}, a_{j}\right)(j=1,2, \ldots, \ell)$ one by one. In this final case, a zigzag graph of $\chi(t)$ with $\chi\left(a_{\ell+1}\right)=0$ gives the result (See Fig.2.2(d)).

Now we continue the proof of Theorem 2.1.2.

Proof of Theorem 2.1.2. The interval $\left(a_{0}, a_{8}\right)$ is separated by 8 sub-intervals $\left(a_{j-1}, a_{j}\right) \quad(j=$ $1,2, \ldots, 8)$. If $f(t)-\varphi(t)$ admits at most 7 zeros, then either $\left(a_{0}, a_{4}\right)$ or $\left[a_{4}, a_{8}\right)$ contains at most 3 zeros. Without loss of generality, we may assume that the interval $\left(a_{0}, a_{4}\right)$ contains at most 3 zeros. By Lemma 2.1 with $g(t)=f(t)-\varphi(t)$ and $k=4$, there exists a continuous function $\chi(t)$ on $\left[a_{0}, a_{4}\right]$ satisfying Lemma 2.2.1 (1). Extend it on $\left(a_{4}, a_{8}\right.$ ] by $\chi(t)=0$. Then we have

$$
\int_{a_{0}}^{a_{8}}(f(t)-\varphi(t)) \chi(t) d t \neq 0,
$$


The Least Number of Intersection Points in Currency Fluctuation and Well- Approximating Line Graph under Constraints of the Elliott Wave Principle

which contradicts to (2.2.2). As a result, $f(t)-\varphi(t)$ must admit at least 8 zeroes.

\subsection{Suggestion}

After making a PC work, the resulting graph of $\varphi(t)$ sometimes possesses less than 8 points of intersection with the graph of $f(t)$. In that case, the minimization of $\operatorname{MSE}_{f}(\vec{a}, \vec{b})$ is possibly attained in the forbidden set - the penalty function $P(\vec{b})$ must be positive. If not, something may be wrong with the algorithm.

\section{References}

[1] Aronson, David R.,2006. "Evidence-Based Technical Analysis, Hoboken, New Jersey, John Wiley and Sons", ISBN 978-0-470-00874-4.

[2] Bryan, Kurt and Shibberu, Yosi. "Penalty Functions and Constrained Optimization", https://www.rose-hulman.edu/ bryan/lottamath/penalt y.pdf\#search $=\% 27$ method + of + penalty + function $\% 27$

[3] Elliott, Ralph Nelson, 1994. "Prechter, Robert R., Jr., ed. R.N. Elliott's Masterworks", Gainesville, GA, New Classics Library, ISBN 978-0-932750-76-1.

[4] Frost, A.J. and Prechter, Robert R. Jr., 2005. "Elliott Wave Principle (10th ed.)", Gainesville, GA, New Classics Library, ISBN 978-0-932750-75-4.

[5] Mandelbrot, Benoit and Richard L. Hudson, 2004. "The (mis)Behavior of Markets", New York: Basic Books, p. 245.

[6] Poser, Steven W.. 2003. "Applying Elliott Wave Theory Profitably. New York, John Wiley and Sons", pp. 2-17., ISBN 978-0-471-42007-1.

[7] Prechter, R., "Elliott Waves, Fibonacci, and Statistics", p. 2., http://www.socionomics.org/pdf/EW Fibo_Statistics.p df\#search=\%27Robert+Prechter+\%282006\%29\%2C+ Elliott+Waves\%2C+Fibonacci\%2C+and+Statistics\%2 $\underline{\mathrm{C} \% 27}$
[8] Wikipedia, "Elliott wave principle", https://en.wikipedia.org/wiki/Elliott_wave_principle

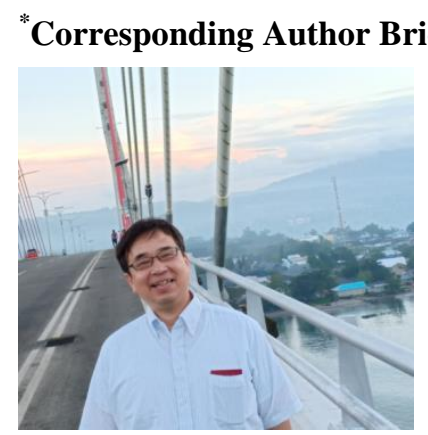

DR. NAOYASU KITA: Faculty Member of Kumamoto University , Faculty of Advanced Science and Technology, Kumamoto University, Science Waseda University (S1), Science Tokyo University (S2), Mathematical Science Nagoya University (S3).

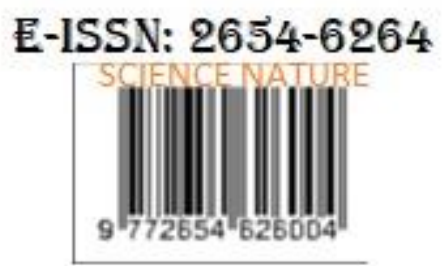

